\title{
Relation between Stochastic Resonance and Synchronization of Passages in a Double-Well System
}

\author{
Mangal C. Mahato and A.M. Jayannavar \\ Institute of Physics, Sachivalaya Marg, Bhubaneswar-751005, India
}

\begin{abstract}
We calculate, numerically, the residence times (and their distribution) of a Brownian particle in a two-well system under the action of a periodic, saw-tooth type, external field. We define hysteresis in the system. The hysteresis loop area is shown to be a good measure of synchronization of passages from one well to the other. We establish connection between this stochastic synchronization and stochastic resonance in the system.
\end{abstract}

PACS numbers: $82.20 . \mathrm{Mj}, 05.40 .+\mathrm{j}, 75.60 . \mathrm{Ej}$

Typeset using REVTEX 
Nature, presumably, exploits the phenomena of stochastic resonance (SR) to its advantage [1,2] to tune in to a desired signal. However, its discovery, initially as a theoretical invention to explain recurrence of ice ages [3, [4], is barely a decade and half old. SR is a nonlinear phenomena wherein an input noise is partially rectified by the system to obtain enhanced output signal at the input signal frequency. It is reflected as a peak in the output signal-to-noise ratio (SNR) as a function of input noise strength. The nature of output signal in the nonlinear system, however, depends on the combined effect of the input signal and the input noise. Since the input signal, in general, is often considered to be weak (subcritical), in the absence of noise no output signal is obtained. The output signal considered is an averaged effect of a large number of realizations of fluctuating forces (noise) in combination with the input signal. The output signal, therefore, may not have the same form as the input signal. However, one expects the output signal to have its dominant time scales of variation nearly synchronous with those of the input signal. The degree of synchronization, however, will depend on the noise strength as well as the input signal parameters. It is, therefore, interesting to study how the output signal gets synchronized with the input signal, and, in particular, whether SR occurs exactly when there is maximum synchronization between the two signals. In this work we show, by calculating the residence time distributions in a periodically forced two-well potential, that there is a close connection between these two phenomena.

We define hysteresis in a two-well system, as explained below, such that its loop area is a good measure of synchronization of passages from one well to the other under the influence of a Gaussian white noise $\hat{f}(t)$ when subjected to an external periodic field $h(t)$. Just in order to make the explanation simpler and more transparent we define hysteresis loop from the distribution of first-passages from one well to the other. ( The explanation may subsequently be simply carried through to the main part of this work wherein residence time distribution is used.) The procedure is illustrated in Figures $1(\mathrm{a}-\mathrm{d}) . \quad \rho(\tau)$ is the distribution of firstpassage-times, $\tau$, from one well to the other (Fig. 1b) obtained by solving the overdamped Langevin equation 


$$
\dot{m}(t)=-\frac{\partial U(m)}{\partial m}+\hat{f}(t)
$$

numerically (for a large number of realisations), where

$$
U(m)=-\frac{a}{2} m^{2}+\frac{b}{4} m^{4}-m h(t)
$$

represents the two-well potential in the presence of an external field $h(t)$ which is periodic in time $t$. The fluctuating forces $\hat{f}(t)$ satisfy: $\langle\hat{f}(t)\rangle=0$ and $\left\langle\hat{f}(t) \hat{f}\left(t^{\prime}\right)\right\rangle=2 D \delta\left(t-t^{\prime}\right)$, where $D$ is the strength of the noise. The sequence of $\tau$ 's is then used to calculate the distribution $\rho(h(\tau))$ (Fig. 1c) of field values $h(\tau)$ at which first-passages occur from the right well to the left one. From $\rho(h(\tau))$ we obtain the upper half of the hysteresis loop $M(h)$ (Fig. 1d),

$$
\frac{M(h)}{h_{c}}=1-2 \int_{h}^{h_{0}} \rho\left(h^{\prime}\right) d h^{\prime}
$$

the other half being obtained by symmetry [5. Here $h_{0}$ is the amplitude and $\left|h_{c}\right|$ is the minimum value of the field $h(t)$ (Fig. 1a) beyond which one of the two wells of $U(m)$ disappears.

From Fig. 1b we see that the peaks of $\rho(\tau)$ occur periodically and centered each time around which $h(t)=-h_{0}$ at which the potential barrier of passage from the right to the left well is the least. Now, perfect synchronization of passages would mean sharp ( $\delta$-function) periodic peaks in $\rho(\tau)$ with periodicity $T_{0}$ of $h(t)$ so that just one sharp peak appears at $h=-h_{0}$ in $\rho(h(\tau))$. This case would yield a rectangular hysteresis loop and hence with the largest possible area. On the other hand, if the passages take place all over randomly, for the other extreme case of least synchronization, $\rho(h(\tau))$ would be uniform resulting in a hysteresis loop of zero area. The hysteresis loop area, thus, provides a measure of the degree of synchronization of passages. It is to be noted that the calculation of hysteresis loop automatically takes into account of informations buried in all the peaks of the passagetime distribution $\rho(\tau)$. It has earlier been shown [5] that hysteresis loop area so calculated acquires a maximum as a function of the input noise strength $D$ as well as a function of the sweep rate $|\dot{h}|$ (equivalent to the frequency) of the saw-tooth type periodic input signal. We 
now discuss our numerical experiment wherein the above explanation about the measure of synchronization should carry through, though not in as obvious a manner, from the residence time distributions in each of the two wells.

In order to obtain the residence time distributions, $\rho_{1}(\tau)$ and $\rho_{2}(\tau)$ in each of the wells 1 and 2, respectively, we monitor the trajectory $m(t)$ of the particle [Eqs. (1) and (2)] for a long time and keep putting markers on the time axis whenever a passage from one well to the other takes place. We take $h(t)$ of the same form as in Fig. 1d but with $h(t=0)=0$. We consider the passage to take place only if the trajectory $m(t)$ crosses the inflexion point on the far side of the maximum of the potential barrier separating the two wells. From the markers on the time axis we also obtain the jump field values $h(t)$ for switching from one well to the other and hence the corresponding distributions $\rho_{12}(h(t))$ and $\rho_{21}(h(t))$. Figures $2 \mathrm{a}$ and $2 \mathrm{~b}$ show the typical plots of $\rho_{1}(\tau)$ and $\rho_{21}(h)$, respectively. Notice that we have, now, calculated $\rho_{12}(h)$, etc., properly so that the distributions spread over the entire period of $h(t)$, and includes both ascending as well as descending parts of $h(t)$. It is now easy to calculate the probability $m_{2}(h)$ that the trajectory lies in the well 2 when the field value $h(t)=h$, from the discrete equation,

$$
m_{2}(h)=m_{2}(h-\Delta h)-m_{2}(h-\Delta h) \rho_{21}(h) \Delta h+m_{1}(h-\Delta h) \rho_{12}(h) \Delta h,
$$

with negligibly small $\Delta h$, and similarly for $m_{1}(h)=1-m_{2}(h)$. We, then, calculate the hysteresis loop $m(h)=m_{2}(h)-m_{1}(h)$ iteratively that satisfies the closed loop condition $m\left(h\left(t+T_{0}\right)\right)=m(h(t))$. Figure 2c shows a typical (asymptotically stationary) hysteresis loop. Please note that the hysteresis loop need not be saturated for every case in contrast to Fig. 1d. We, now, discuss the results on the stochastic synchronization(SS) of passages from the variation of hysteresis loop area as a function of noise strength $D$ and also as a function of sweep rate $|\dot{h}|$.

Throughout our calculation, we take $a=2.0$ and $b=1.0$ in the expression for $U(m)$, so that the barrier height when $h(t)=0$ is 1 . We take $h_{0}=.7 h_{c}$ and $.9 h_{c}$ and calculate for each of these cases, the distributions $\rho_{1}(t)$ and $\rho_{2}(t)$ and also the passage field distributions 
$\rho_{12}(h(t))$ and $\rho_{21}(h(t))$ for various values of $\dot{h}$. The results obtained for both the field amplitudes $0.7 h_{c}$ and $0.9 h_{c}$ have qualitatively similar trends. Figure 3 shows the variation of hysteresis loop area as a function of noise strength $D$. We observe that the hysteresis loop area initially increases as D is increased from a small value, attains a maximum value at $D=D_{m a}$, say, and then decreases gradually as $D$ is increased further. It shows that at $D=D_{m a}$ the output signal is most synchronized with the input signal $h(t)$. The hysteresis loop area is also found to show maximum as a function of $h$ (Fig. 3b).

From the residence time distributions we calculate the Fourier transform and each of the components are squared to obtain the power spectral density [6]. As expected large peaks are obtained at regular intervals. We calculate the ratio of the height of the first peak and the (background) noise level at the same frequency. This signal-to-noise ratio (SNR) is plotted in Fig. 4 as a function of $D$. The errors in calculating the SNR are large mostly because of the arbitrariness in fixing the (low) background noise level. However, the errors do not affect the general trends of our results. We, indeed, find SR in the usual sense of the SNR maxima. Interestingly, the value of $D=D_{S N R}(\dot{h})$ at which SR occurs, is not the same as the value of $D=D_{m a}(\dot{h})$ at which the passages are the most synchronized. However, $D_{S N R}(\dot{h})$ and $D_{m a}(\dot{h})$ are quite close and they tend to become closer as $\dot{h} \rightarrow 0$, as shown in Fig. 5. (A finer examination to smaller $\dot{h}$ is beyond the computing power available to us.) SR and SS are, thus, not unrelated [0,8]. SR may, therefore, be a natural manifestation of SS. Moreover, SR, defined as the maximum of SNR appears not only as a function of noise strength $D$ but also as a function of the sweep rate $\dot{h}$ (or equivalently the frequency) of the external field (input signal) as shown in Fig. 6 (cf. with Fig. 3b).

In conclusion we state that hysteresis loop area, which is an average effect of all the peaks in the residence time distribution, is a good measure of synchronization of passages with the input signal. In an earlier work [7], by taking the only first few peaks, and in particular the first peak, of the residence time distribution, SR was given an alternative description and was shown to be a bonafide resonance. On closer examination we find that rest of the peaks 
too play important role. We, however, concretise their assertion, by taking into account all the peaks of the residence time distribution through the hysteresis loop area, that SR is a genuine resonance, at least in a double-well potential system, and is due to the synchronized response of the system to the input periodic signal. Also, the degree of synchronization of passages and the SNR show maximum as a function of the sweep rate (or equivalently the frequency) of the input signal [9]. 


\section{REFERENCES}

[1] See, for example, Proceedings of the NATO ARW Stochastic Resonance in Physics and Biology, edited by F. Moss, A. Bulsara, and M.F. Shlesinger [J. Stat. Phys. 70, No. 1 (1993)]

[2] J.K. Douglass, L. Wilkens, E. Pantazelou, and F. Moss, Nature (London) 365, 337 (1993).

[3] A.R. Benzi, A. Sutera, and A. Vulpiani, J. Phys. A: Math. Gen. 14, L453 (1981).

[4] B. McNamara and K. Wiesenfeld, Phys. Rev. A 39, 4854 (1989); P. Jung, Phys. Rep. 234, 175 (1993) and references therein.

[5] M.C. Mahato and S.R. Shenoy, Phys. Rev. E 50, 2503 (1994).

[6] W.H. Press, S.A. Teukolsky, W.T. Vetterling, and B.P. Flannery, Numerical Recipes (in Fortran):The Art of Scientific Computing, Cambridge Univ. Press, 1992, pp. 542.

[7] L. Gammaitoni, F. Marchesoni, and S. Santucci, Phys. Rev. Lett. 74, 1052 (1995).

[8] A.R. Bulsara and L. Gammaitoni, Phys. Today, 49, 39 (March 1996).

[9] Similar results are obtained analytically recently by V. Berdichevsky and M. Gitterman, J. Phys. A: Math. Gen. 29, L447 (1996). 
FIG. 1. Shows (a) the time variation of the external field $h(t)$, (b) the first-passage-time distribution $\rho(\tau)$, (c) the passage field distribution $\rho(h)$, and (d) the corresponding hysteresis loop calculated from $\rho(h)$, for $h_{0}=0.7 h_{c}, D=0.3$, and the period of $h(t), T_{0}=28.0$.

FIG. 2. Shows (a) the residence time distribution $\rho_{1}(\tau)$ in the well 1 , (b) the passage field distribution $\rho_{21}(h)$ for passage from well 2 to well 1 , and (c) the corresponding hysteresis loop $m(h)$ for $h_{0}=0.9 h_{c}, D=0.2$, and period of $h(t), T_{0}=36.0$.

FIG. 3. Plots of (a) hysteresis loop area $A$ as a function of $D$ for $\dot{h}=0.05 h_{c}(\circ), 0.1 h_{c}(\square)$, $0.2 h_{c}(\diamond), 0.4 h_{c}(\triangle)$, and $0.6 h_{c}(\nabla)$, and (b) hysteresis loop area $A$ as a function of field sweep rate $\dot{h}$ for $D=0.1(\circ), 0.15(\square), 0.2(\diamond)$, and $0.3(\triangle)$ for $h_{0}=0.9 h_{c}$. In this and in the rest of the figures the lines joining the points are only to guide the eye.

FIG. 4. Plots of signal-to-noise ratio (SNR) as a function of $D$, for $\dot{h} / h_{c}=0.05(\circ), 0.1(\square)$, $0.2(\diamond), 0.4(\triangle), 0.5(\triangleleft), 0.6(\nabla)$, and $0.72(\triangleright)$, for $h_{0}=0.9 h_{c}$.

FIG. 5. Plots the peak positions of the plots of hysteresis loop area $A$ versus $D(\circ)$ and those of SNR versus $D(\square)$, for $h_{0}=0.7 h_{c}$ (empty symbols, solid joining lines) and for $h_{0}=0.9 h_{c}$ (filled symbols, dashed joining lines).

FIG. 6. Shows SNR as a function of $\dot{h}$ for $h_{0}=0.9 h_{c}$, for various values of $D=0.1(\circ), 0.15(\square)$, and $0.2(\diamond)$. 